\title{
Die klinisch-forensische Bedeutung des Selbstregulationsmodells sexueller Delinquenz und Rückfälligkeit bei pädosexuellen Straftaten
}

\author{
Priscilla Gregório Hertz ${ }^{1}$ Martin Rettenberger ${ }^{2,3}$ • Robin Welsch ${ }^{4} \cdot$ Daniel Turner $^{1}$
}

Eingegangen: 2. Juni 2020 / Angenommen: 12. Juni 2020 / Online publiziert: 27. Juli 2020

(C) Der/die Autor(en) 2020

\section{Zusammenfassung}

Das Selbstregulationsmodell sexueller Rückfälligkeit (,self-regulation model of the relapse process“, SRM) stellt eine Theorie zur Ätiologie sexueller Delinquenz und Rückfälligkeit dar, bei der sexuell motivierte Straftaten und Täter unterschiedlichen Rückfallpfaden und Entscheidungswegen zugeordnet werden, von denen aus wiederum auf individuelle Motive, Defizite und Ressourcen geschlossen werden kann. In der vorliegenden Studie wurde eine Stichprobe von $N=68$ Männern, die mindestens eine sexuell motivierte Straftat gegen Kinder begangen haben, in Bezug auf die SRM-Typologisierung beurteilt und hinsichtlich verschiedener klinischer, demografischer und kriminologischer Merkmale verglichen. Unter Verwendung des SRM konnten $25 \%(n=17)$ dem annähernd-expliziten, $25 \%(n=17)$ dem vermeidend-passiven, $22,1 \%(n=15)$ dem annähernd-automatischen und 13,2\% $(n=9)$ dem vermeidend-aktiven Rückfallpfad zugeordnet werden. Männer mit Annäherungszielen wiesen im Vergleich zu denen mit Vermeidungszielen höhere Werte im Static-99 und ein entsprechend höheres Rückfallrisiko auf. Außerdem wurde bei Männern mit Annäherungszielen häufiger die Diagnose einer Pädophilie gestellt. Die Ergebnisse liefern erste Hinweise dafür, dass das SRM ein nützliches theoretisches Modell sein kann, um Gemeinsamkeiten zwischen Männern, die aufgrund eines Kindesmissbrauchsdelikt verurteilt wurden, aufzudecken.

Schlüsselwörter Sexualstraftäter $\cdot$ Kindesmissbrauch $\cdot$ Selbstregulation $\cdot$ Pädophilie $\cdot$ Static-99

Priscilla Gregório Hertz wurde für diese Arbeit auf dem 9. Symposium „Empirische Forschung in der Forensischen Psychiatrie, Psychologie und Psychotherapie (EFPPP)“ in Mainz am 12. und 13.09.2019 mit dem Eberhard-Schorsch-Preis der Deutschen Gesellschaft für Sexualforschung (DGfS) ausgezeichnet.

Priscilla Gregório Hertz priscilla.gregorio_hertz@unimedizin-mainz.de
1 Klinik für Psychiatrie und Psychotherapie, Universitätsmedizin Mainz, Untere Zahlbacherstraße 8, 55131 Mainz, Deutschland

2 Kriminologische Zentralstelle (KrimZ), Wiesbaden, Deutschland

3 Psychologisches Institut, Johannes Gutenberg Universität Mainz (JGU), Mainz, Deutschland

4 Human-Centered Ubiquitous Computing, Ludwig-Maximilians-Universität, München, Deutschland 


\title{
The clinical and forensic relevance of the self-regulation model of sexual delinquency and recidivism in pedosexual offenses
}

\begin{abstract}
The self-regulation model of the offense and relapse process (SRM) represents a theoretical approach to explain and assess the process of sexual delinquency and recidivism. According to this model, sexual offenses and offenders can be categorized into four different recidivism pathways, which are described by individual motives, deficits and resources recognized throughout the relapse process. In the present study, a sample of $N=68$ men who were convicted of at least 1 sexual offense against child victims were assessed regarding the offense pathways of the SRM and compared with respect to clinical, demographic and criminological features. The results showed that $25 \%(n=17)$ of the sample were allocated to the approach-explicit, 25\% $(n=17)$ to the avoiding-passive, 22.1\% $(n=15)$ to the approach-automatic, and $13.2 \%(n=9)$ to the avoiding-active pathway. Men with approach goals showed higher scores in Static-99 and therefore a higher recidivism risk for sexual offenses than men with avoidance goals. Furthermore, men with an approach goal were more often diagnosed with a pedophilic disorder. The results of the present study provide first indicators that the SRM can be a useful theoretical model to detect similarities between men who were convicted of sexual abuse crimes with children.
\end{abstract}

Keywords Sexual offender $\cdot$ Sexual abuse $\cdot$ Self-regulation $\cdot$ Pedophilia $\cdot$ Static-99

\section{Einleitung}

Personen, die aufgrund einer Sexualstraftat verurteilt wurden, stellen eine heterogene Gruppe dar und unterscheiden sich u. a. bezüglich ihrer persönlichen Ziele, ihrer kognitiven Möglichkeiten, die Taten zu planen, sowie bezüglich der Emotionen, die vor, während und nach der Sexualstraftat erlebt wurden (Marshall 1996; Ward et al. 1995; Yates und Ward 2007, 2008). Die Aufteilung dieser Gesamtpopulation in möglichst homogene Gruppen stellt wiederum eine Voraussetzung dafür dar, spezifische und wirksame Therapie- und Interventionsmöglichkeiten für diese Gruppe bzw. mögliche Untergruppen zu entwickeln (Turner und Rettenberger 2020).
Das Selbstregulationsmodell sexueller Rückfälligkeit (,self-regulation model of the relapse process", SRM; Ward und Hudson 1998, 2000) ist ein theoretisches Modell zur Untergliederung und Einteilung von Männern, die bereits einmal aufgrund einer Sexualstraftat verurteilt wurden. Das SRM kategorisiert sexuelle (Rückfall-)Delikte hinsichtlich a) des auslösenden Ereignisses oder der auslösenden Faktoren, b) der Ziele, die mit der Sexualstraftat verfolgt werden, c) des Ausmaßes an planendem Verhalten und des Einsatzes gezielter Strategien zu Auswahl und Überwältigung der Opfer sowie d) der affektiven Bewertung des sexuellstraffälligen Verhaltens nach dem sexuellen Übergriff. Das SRM umfasst hierbei kognitive, verhaltensbezogene, affektive und kontextbezogene Faktoren (Ward und Hudson

Tab. 1 Zusammenfassende Beschreibung der 4 Tatbegehungspfade nach dem SRM. (Ward und Hudson 1998, 2000)

\begin{tabular}{ll}
\hline Vermeidend- & Bei Tätern, die dem vermeidend-passiven Pfad folgen, werden die persönlichen Coping-Mechanismen durch ein Ereignis mit \\
passiv & hoher individueller Belastung im Tatvorfeld überfordert. Daraufhin entsteht ein Verlangen, die negativen Gefühle, die mit der \\
& Überforderung einhergehen, durch deviante sexuelle Verhaltensweisen zu reduzieren, wobei der Täter dies auf eine passive Art \\
& und Weise macht. Durch den unterregulierten Selbstregulationsstil wird in einer Hochrisikosituation dem sexuellen Verlangen \\
& nachgegeben, und es kommt aufgrund des Kontrollverlusts zur Straftat. Der Täter erlebt sich danach tendenziell eher defizitär \\
& und beschämt
\end{tabular}

Vermeidend- $\quad$ Auch bei diesem Pfad werden die Coping-Mechanismen durch ein Belastungsereignis im Tatvorfeld überfordert. Der Täter aktiv versucht jedoch, mit aktiven, aber dysfunktionalen Coping-Strategien dagegenzusteuern (fehlreguliert). Die gewählten Selbstregulationsstrategien sind ineffektiv und wirken kontradiktorisch, d.h., erhöhen das Risiko (z. B. Substanzkonsum) und führen zu Kontrollverlusterleben und zur Straftat. Nach der Straftat erlebt sich der Täter eher schuldig als beschämt

Annähernd- Im annähernd-automatischen Pfad überwiegen automatisierte Verhaltensskripts aus früheren Taten oder eine ausgeprägte Fanautomatisch tasietätigkeit sowie ein unterregulierter Selbstregulationsstil. Der Täter begibt sich in eine Hochrisikosituation, wobei dies eher zufällig als geplant geschieht. Diese Hochrisikosituation triggert die Aktivierung des devianten Verhaltensskripts und führt zur Straftat. Typischerweise erscheinen diese Taten wie ,aus heiterem Himmel“ kommend und sind in ihrer Dauer tendenziell sehr kurz. Anders als bei den beiden Vermeidungspfaden erleben die Täter im annähernd-automatischen Pfad einen positiven Affekt bei negativen Einstellungen gegenüber dem Opfer

Annähernd- In diesem Pfad beruht die Begehung von Sexualstraftaten auf bewusster, strategischer Planung vor dem Hintergrund des stark explizit ausgeprägten Ziels, sexuelle Gratifikation durch deviantes sexuelles Verhalten zu erreichen. Dabei liegen intakte d.h. effektive Selbstregulationsstrategien vor. Zusätzlich zum positiven Affekt nach der Tat erfolgen bei dem Täter eine Verfeinerung und ein Ausbau der angewandten Strategien 
1998, 2000). Männer, die aufgrund einer Sexualstraftat verurteilt wurden, lassen sich, basierend auf diesem Modell und einem 9-stufigen Entscheidungsbaum, 4 verschiedenen Kategorien bzw. Rückfallpfaden zuordnen, die sich nach dem verfolgten Ziel im Hinblick auf die Sexualstraftatbegehung und den angewendeten Strategien unterscheiden (für weitere Details: Ward und Hudson 1998, 2000). Dabei werden zum einen Vermeidungs- und Annäherungsziele im Hinblick auf die sexuelle Straftat unterschieden, und zum anderen können die angewandten Selbstregulationsstrategien in aktiv/explizit oder passiv/automatisch unterschieden werden. Es wird angenommen, dass die verschiedenen Delikt- und Rückfallpfade auf unterschiedliche Bedürfnisse der Männer, die aufgrund einer Sexualstraftat verurteilt wurden, zurückgeführt werden können, von denen sich wiederum Hinweise auf die individuellen Motive, Ressourcen und Defizite ableiten lassen. Einen zusammenfassenden Überblick über die 4 im SRM vorgeschlagenen Rückfallpfade gibt Tab. 1.

\section{Empirische Fundierung}

In früheren Untersuchungen, in denen das SRM angewandt wurde, konnte gezeigt werden, dass Personen, die vorrangig Annäherungsziele verfolgten, mehr kognitive Verzerrungen bezüglich sexueller Kontakte mit Kindern und eine höhere emotionale Kongruenz mit Kindern zeigten sowie häufiger extrafamiliäre und männliche Opfer aufwiesen, im Vergleich zu Männern, die einem der beiden vermeidenden Tatbegehungspfade zugeordnet werden konnten (Bickley und Beech 2002). Darüber hinaus wiesen Männer mit einem Annäherungsziel auch kriminologische Auffälligkeiten auf, wie z.B. eine höhere Opferzahl, mehr Gewaltanwendung während des Indexdelikts sowie eine höhere Zahl an früheren Anklagen und Verurteilungen aufgrund von Sexualstraftaten (Kingston et al. 2012). Außerdem wurden bei Männern, die aufgrund eines Sexualdelikts gegen Kinder oder Erwachsene verurteilt wurden und dem annäherndautomatischen Tatbegehungspfad zugeordnet werden konnten, mehr Impulskontrolldefizite und psychopathische Züge im Vergleich zu den anderen 3 Gruppen gefunden (Gotch et al. 2007; Kingston et al. 2009, 2012; Leguizamo et al. 2010; Simons et al. 2008; Webster 2005). Täter mit Vermeidungszielen hatten hingegen häufiger ausschließlich weibliche und intrafamiliäre Opfer und waren zum Zeitpunkt der Straftat häufiger in lange bestehenden Beziehungen und hatten eigene Kinder (Bickley und Beech 2002; Yates und Kingston 2006).

Im Hinblick auf die angewandten Selbstregulationsstrategien konnte in früheren Untersuchungen festgestellt werden, dass Täter mit passiven bzw. automatischen Strategien die Schuld der Straftat eher äußeren Umständen zuschrieben und mehr frühere Verurteilungen aufgrund von Sexual- straftaten aufwiesen (Bickley und Beech 2002). Demgegenüber war bei Männern mit expliziten oder aktiven Selbstregulationsstrategien eine höhere Vielfalt bezüglich der Opferauswahl zu beobachten, d.h., diese Männer hatten häufig sowohl kindliche als auch erwachsene Opfer (Yates und Kingston 2006).

Neben diesen kriminologisch relevanten Faktoren variierten auch die statischen und dynamischen Risikofaktoren in Abhängigkeit von den 4 Tatbegehungspfaden (Yates und Kingston 2006). So wiesen Probanden, die ein Annäherungsziel verfolgten (v.a. annähernd-explizit) signifikant höhere Werte im Static-99 auf (Hanson und Thornton 1999; Rettenberger und Eher 2006), im Vergleich zu Männern mit einem Vermeidungsziel (Yates und Kingston 2006). Der Static-99-Gesamtwert war darüber hinaus in der Lage vorherzusagen, welchem Pfad ein individueller Sexualstraftäter zugeordnet werden konnte, d.h., je höher der Static-99-Gesamtwert, umso wahrscheinlicher war die Zuordnung zu einem der Annäherungspfade ( $O R=1,7$; Yates und Kingston 2006). Des Weiteren wiesen Täter mit Annäherungszielen auch mehr dynamische Risikofaktoren auf, woraus nicht nur auf ein höheres Risiko, sondern auch auf einen höheren Therapiebedarf dieser Tätergruppe geschlossen werden konnte (Kingston et al. 2012). Gleichzeitig scheinen Männer mit Annäherungszielen (insbesondere aus dem annähernd-expliziten Pfad) nur wenig von psychotherapeutischen Interventionen zu profitieren (Webster 2005).

In der bisher umfangreichsten Untersuchung zum SRM wurden die Behandlungsergebnisse sowie Rückfallraten in einer prospektiven Studie anhand einer Stichprobe von 275 Männern, die aufgrund einer Sexualstraftat verurteilt wurden, innerhalb eines durchschnittlichen Nachbeobachtungszeitraums von 4 Jahren $(S D=1,55)$ nach Behandlung untersucht (Kingston et al. 2013). Hierbei wurden die Werte im Stable-2000 (Hanson und Harris 2001; Rettenberger et al. 2011) sowie die selbst angegebenen Behandlungsziele vor und nach der Behandlung miteinander verglichen, wobei für soziale Erwünschtheit und den Static-99-Wert kontrolliert wurde. Unabhängig vom Tatbegehungspfad zeigte sich bei allen Sexualstraftätern nach der Behandlung eine Reduktion der dynamischen Risikofaktoren (Kingston et al. 2013). Dennoch wurde bei Männern, die dem annäherndautomatischen Pfad folgten, eine höhere Rate an gewalttätiger (inklusive sexueller) Rückfälligkeit im Vergleich zu den anderen 3 Gruppen gefunden.

Untersuchungen zur Anwendung des SRM im deutschsprachigen Raum fehlen bisher. In der vorliegenden Studie wurde das SRM in Anlehnung an die oben dargestellten Forschungsergebnisse in einer Stichprobe von Männern, die aufgrund eines Kindesmissbrauchsdelikts verurteilt wurden, hinsichtlich demografischer, klinischer und straftatbezogener Merkmale sowie hinsichtlich des stati- 
schen Rückfallrisikos untersucht. Es wurde dabei insbesondere erwartet, dass das Rückfallrisiko bei Männern mit einem Annäherungsziel höher ausfallen würde als bei Personen mit einem Vermeidungsziel (z. B. Kingston et al. 2012, 2013).

\section{Methode}

\section{Stichprobe}

Insgesamt konnten $N=68$ Probanden in die vorliegende Untersuchung eingeschlossen werden. Die Teilnehmer der aktuellen Untersuchung wurden in verschiedenen Einrichtungen des Maßregel- und des Justizvollzugs in Deutschland rekrutiert. Einschlusskriterien waren hierbei: männliches Geschlecht, mindestens 18 Jahre alt, mindestens eine frühere Verurteilung aufgrund eines sexuellen Kindesmissbrauchsdelikts (ausschließlich „Hands-on“- oder Kontaktdelikte) und ausreichende Kenntnisse der deutschen Sprache. Ausgeschlossen wurden Männer, die schwere zentralneurologische Erkrankungen aufwiesen, und solche, die ausschließlich aufgrund des Besitzes oder Konsums kinderpornografischen Materials verurteilt wurden.

Das Durchschnittsalter zum Zeitpunkt der Erhebung lag bei $M=44,6(S D=11,9)$ Jahren und bei der ersten strafrechtlichen Auffälligkeit bei $M=27,7(\mathrm{SD}=12,8)$ Jahren. Die Teilnehmer hatten durchschnittlich $M=9,3(S D=1,6)$ Jahre Schulausbildung absolviert. Hinsichtlich des Familienstands zum Zeitpunkt des Delikts waren 48,5\% $(n=33)$ ledig, 19,1\% $(n=13)$ verheiratet oder in einer Partnerschaft lebend, $25 \%(n=17)$ geschieden und 2,9\% $(n=2)$ verwitwet.

Die Länge der aufgrund des Indexdelikts verhängten Freiheitsstrafe über alle Teilnehmer hinweg betrug im Durchschnitt $M=4,5$ Jahre $(S D=2,6)$. Durchschnittlich wiesen die Teilnehmer $M=2,5(S D=3,6)$ Vorstrafen auf (Gewaltdelikte: $M=0,2, S D=0,5$; Sexualdelikte $M=0,8$, $S D=1,6)$. Des Weiteren hatten 30,9\% $(n=21)$ der Teilnehmer ausschließlich intrafamiliäre Opfer, und bei $58,8 \%$ $(n=40)$ war das Opfer dem Täter bereits vor der Straftat bekannt. Insgesamt $60,3 \%(n=41)$ der Täter hatten lt. Akten die sexuelle Straftat zugegeben, während 22,1\% $(n=15)$ als Tatleugner eingestuft wurden.

\section{Datenerhebung}

Die vorliegende Untersuchung erhielt durch die Ethikkommission der Psychotherapeutenkammer Hamburg ein positives Votum. Sie war Teil eines umfangreichen Projekts zur Untersuchung neuropsychologischer und neurokognitiver Auffälligkeiten von Sexualstraftätern (z. B. Turner et al. 2018). Im Rahmen des Projekts wurden alle Teilnehmer gebeten, verschiedene Fragebogen zum Thema Sexualität auszufüllen und an 2 Testtagen verschiedene Verhaltensparadigmen an einem Computer durchzuführen (Turner et al. 2018; Welsch et al. 2020 für weitere Ergebnisse). Pro Testtag betrug die Untersuchungsdauer etwa 90Min. Die Probanden erhielten für ihre Teilnahme entweder $20 €$ in bar ausbezahlt oder in Form eines Telefongutscheins.

\section{Messinstrumente}

\section{Protokoll zur Bewertung des Self-regulation model}

Die Einschätzung der Probanden hinsichtlich der SRM-Pfade wurde retrospektiv von einem geschulten und erfahrenen Rater mithilfe eines semistrukturierten Protokolls durchgeführt (für weitere Details: Yates et al. 2009). Das Protokoll enthielt insgesamt 7 Item-Bereiche, die in 2 übergeordnete Kategorien eingeteilt werden konnten: (a) Ziele im Zusammenhang mit den Straftaten (bestehender Wunsch, die Straftaten zu verhindern, Einstellung zu den Straftaten, kognitive Verzerrungen und Bewertung der Straftaten nach der Begehung der Straftaten) und (b) angewandte Strategien bei Begehung der Straftaten (Selbstregulationsfähigkeit, Planung und wahrgenommene Kontrolle über das straffällige Verhalten). Hierbei enthielt das angewendete Protokoll eine detaillierte Liste von Indikatoren, die auf das Vorhandensein eines Annäherungs- oder Vermeidungsziels sowie bezüglich der angewandten Selbstregulationsstrategien hindeuteten. Das Protokoll wurde in früheren Untersuchungen an unabhängigen Stichproben von Sexualstraftätern validiert und weist nachweislich eine hohe Reliabilität auf (Kingston et al. 2009; Leguizamo et al. 2010). Die zur Bewertung des Protokolls verwendeten Informationen wurden unabhängig von den Ergebnissen der verwendeten Tests und Prognoseinstrumenten erhoben.

\section{Static-99}

Zur Risikoeinschätzung wurde der Static-99 (Hanson und Thornton 2000; Rettenberger und Eher 2006) verwendet, ein international etabliertes aktuarisches Prognoseinstrument zur Einschätzung des Rückfallrisikos für sexuell motivierte Straftaten. Der Static-99 beinhaltet 10 ausschließlich statische Risikofaktoren, d.h. solche, die per Definition nicht veränderbar sind. Der Static-99 wurde basierend auf den Informationen, die aus den Vollzugsheften der Probanden gewonnen werden konnten, kodiert.

\section{Statistische Analysen}

Zunächst wurde die Häufigkeit der einzelnen Tatbegehungspfade nach dem SRM (Ward und Hudson 1998, 2000) in der vorliegenden Stichprobe bestimmt. Darüber hinaus wur- 
den Unterschiede bezüglich der demografischen Variablen Alter zum Erhebungszeitpunkt, Anzahl der eigenen Kinder und Schuljahre der Probanden in Abhängigkeit von den 4 Pfaden untersucht. Zudem wurden Gruppenunterschiede in den kriminologischen Merkmalen Alter bei erster Auffälligkeit, Anzahl der Vorstrafen, Vorstrafen mit einem Sexualdelikt, Anzahl der Opfer und Höhe der Freiheitsstrafe berechnet. Da frühere Untersuchungen zeigten, dass insbesondere der Vergleich zwischen Annäherungs- vs. Vermeidungszielen sowie aktiven/expliziten vs. passiven/ automatischen Selbstregulationsstrategien von Bedeutung sein könnte, wurden die folgenden Vergleiche zwischen diesen beiden Gruppen durchgeführt (z. B. Bickley und Beech 2002). Zunächst wurden die gefundenen Gruppen bezüglich klinischer Merkmale (Ort der Unterbringung, psychiatrische Diagnosen) untersucht. Im letzten Schritt wurden die gefundenen Gruppen in Bezug auf Unterschiede im Static-99-Gesamtwert untersucht. Die statistischen Analysen wurden mittels SPSS (Statistical Package for the Social Sciences; 23v) durchgeführt.

\section{Ergebnisse}

Von den eingeschlossenen $N=68$ Probanden konnten 47,1\% ( $n=32)$ einem Annäherungs- und 38,2\% ( $n=26)$ einem Vermeidungsziel zugeordnet werden, während bei $10,3 \%(n=7)$ der Probanden keine eindeutige Zuordnung vorgenommen werden konnte (Tab. 2). Insgesamt 39,7\% $(n=27)$ der Probanden wählten aktive oder explizite Selbstregulationsstrategien zur Delikt- bzw. zur Rückfallvermei- dung, während 52,9\% $(n=36)$ passive bzw. automatische Strategien verwendeten. Es konnte 7,3\% $(n=5)$ der Probanden keine eindeutige Selbstregulationsstrategie zugeordnet werden.

\section{Gruppenunterschiede}

Der Vergleich der demografischen und kriminologisch-relevanten Daten in Abhängigkeit vom Tatbegehungspfad ergab, dass Männer, die dem vermeidend-aktiven Pfad zugeordnet werden konnten, eine signifikant längere Schulausbildung aufwiesen als Probanden im vermeidend-passiven Pfad (Wilks' $\lambda=3,809, F(3,53), p=0,015$; Tab. 3).

Darüber hinaus wurden Personen in den beiden Annäherungspfaden zu signifikant längeren Freiheitsstrafen infolge des Indexdelikts verurteilt, im Vergleich zu Personen aus den beiden Vermeidungspfaden. Männer, die dem annähernd-automatischen Pfad zugeordnet werden konnten, waren außerdem signifikant jünger bei der ersten strafrechtlichen Auffälligkeit als die Männer im vermeidend-aktiven Pfad, Wilks' $\lambda=2,075, F(15,127), p=0,015$ (Tab. 4).

Tab. 2 Häufigkeit der einzelnen Tatbegehungspfade nach dem SRM. (Ward und Hudson 1998, 2000)

\begin{tabular}{ll}
\hline Vermeidend-passiv & Vermeidend-aktiv \\
\hline $25 \%$ & $13,2 \%$ \\
$(n=17)$ & $(n=9)$ \\
Annähernd-automatisch & Annähernd-explizit \\
$22,1 \%$ & $25 \%$ \\
$(n=15)$ & $(n=17)$ \\
\hline
\end{tabular}

Tab. 3 Vergleich demografischer Variablen in Abhängigkeit vom Tatbegehungspfad

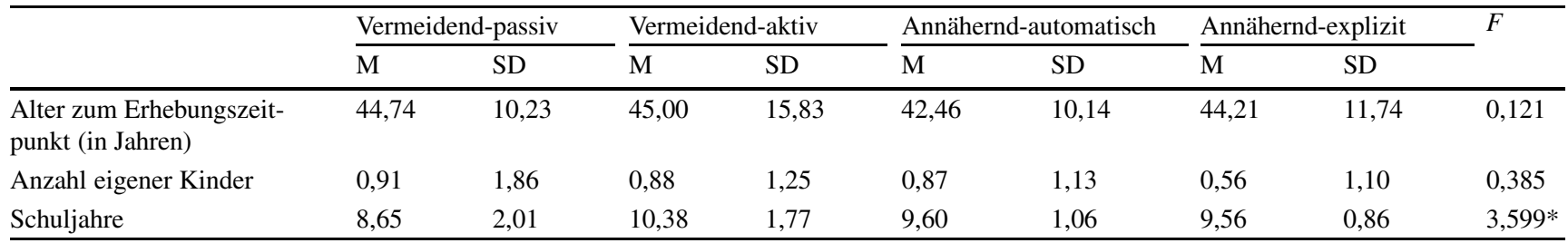

Wilks' $\lambda=0,216, F(3,53)=3,809, * p<0,05$

Tab. 4 Vergleich kriminologischer Variablen in Abhängigkeit vom Rückfallpfad

\begin{tabular}{|c|c|c|c|c|c|c|c|c|c|}
\hline & \multicolumn{2}{|c|}{ Vermeidend-passiv } & \multicolumn{2}{|c|}{ Vermeidend-aktiv } & \multicolumn{2}{|c|}{ Annähernd-automatisch } & \multicolumn{2}{|c|}{ Annähernd-explizit } & \multirow[t]{2}{*}{$F$} \\
\hline & $\mathrm{M}$ & SD & M & SD & M & SD & $\mathrm{M}$ & SD & \\
\hline $\begin{array}{l}\text { Alter bei erster Auffäl- } \\
\text { ligkeit }\end{array}$ & 26,55 & 12,19 & 40,71 & 15,58 & 22,73 & 10,73 & 30,25 & 11,16 & $3,505^{*}$ \\
\hline Anzahl der Vorstrafen & 3,65 & 4,18 & 1,00 & 1,92 & 3,64 & 4,91 & 1,94 & 2,67 & 1,347 \\
\hline $\begin{array}{l}\text { Vorstrafen mit Sexual- } \\
\text { delikt }\end{array}$ & 1,02 & 2,41 & 0,29 & 0,76 & 0,55 & 0,69 & 1,05 & 1,53 & 0,483 \\
\hline Anzahl der Opfer & 1,85 & 1,27 & 3,57 & 3,16 & 1,64 & 1,57 & 3,00 & 2,19 & 2,479 \\
\hline $\begin{array}{l}\text { Höhe der Freiheitsstra- } \\
\text { fe }\end{array}$ & 3,32 & 1,57 & 3,57 & 1,86 & 5,73 & 2,58 & 5,63 & 3,04 & $4,348 * *$ \\
\hline
\end{tabular}

Wilks' $\lambda=0,547, F(15,127)=2,075, * * p<0,001, * p<0,05$ 
Tab. 5 Vergleich klinischer Variablen in Abhängigkeit vom Tatbegehungspfad

\begin{tabular}{llll}
\hline & Annäherungsziel & Vermeidungsziel & $p$ \\
\hline Keine Diagnose & $5(15,5 \%)$ & $3(11,5 \%)$ & 0,94 \\
Pädophilie & $25(78,1 \%)$ & $13(50,0 \%)$ & 0,05 \\
Persönlichkeitsstörung & $8(25,0 \%)$ & $9(34,6 \%)$ & 0,61 \\
Angst- und affektive Störungen & $1(3,1 \%)$ & $5(19,2 \%)$ & 0,12 \\
Suchterkrankungen & $4(12,5 \%)$ & $3(11,5 \%)$ & 0,77 \\
Maßregelvollzug & $12(37,5 \%)$ & $8(30,8 \%)$ & 0,79 \\
Justizvollzug & $14(43,8 \%)$ & $3(11,5 \%)$ & 0,02 \\
Nachsorgeambulanz & $6(18,8 \%)$ & $15(57,7 \%)$ & 0,01 \\
- & Passiv/automatisch & Aktiv/explizit & - \\
Keine Diagnose & $4(11,1 \%)$ & $5(18,5 \%)$ & 0,64 \\
Pädophilie & $23(63,9 \%)$ & $15(55,6 \%)$ & 0,50 \\
Persönlichkeitsstörung & $10(27,8 \%)$ & $7(25,9 \%)$ & 0,87 \\
Angst- und affektive Störungen & $4(11,1 \%)$ & $3(11,1 \%)$ & 0,99 \\
Suchterkrankungen & $6(16,7 \%)$ & $3(11,1 \%)$ & 0,53 \\
Maßregelvollzug & $14(38,9 \%)$ & $6(22,2 \%)$ & 0,16 \\
Justizvollzug & $9(25,0 \%)$ & $10(37,0 \%)$ & 0,30 \\
Nachsorgeambulanz & $11(30,6 \%)$ & $11(40,7 \%)$ & 0,40 \\
\hline
\end{tabular}

Tab. 6 Vergleich des Rückfallrisikos in Abhängigkeit vom Tatbegehungspfad

\begin{tabular}{|c|c|c|c|c|c|}
\hline \multirow{2}{*}{$\begin{array}{l}\text { Ziel } \\
\text { Static-99 (Risikokategorie) }\end{array}$} & \multicolumn{2}{|c|}{ Vermeidungsziel } & \multicolumn{3}{|c|}{ Annäherungsziel } \\
\hline & $\mathrm{M}$ & $\mathrm{SD}$ & $\mathrm{M}$ & $\mathrm{SD}$ & $F$ \\
\hline & 1,17 & 0,98 & 1,71 & 0,86 & $4,511 *$ \\
\hline Selbstregulationsstrategien & \multicolumn{2}{|c|}{ Passiv/automatisch } & \multicolumn{3}{|c|}{ Aktiv/explizit } \\
\hline \multirow[t]{2}{*}{ Static-99 (Risikokategorie) } & M & SD & M & SD & $F$ \\
\hline & 1,38 & 0,98 & 1,50 & 0,95 & 0,241 \\
\hline
\end{tabular}

${ }^{*} p<0,05$
Des Weiteren wiesen Männer mit einem Annäherungsziel signifikant häufiger eine Pädophiliediagnose auf und befanden sich überwiegend im Justizvollzug. Zudem wiesen Männer mit einem Annäherungsziel ein höheres statisches Rückfallrisiko auf (gemessen mit dem Static-99, Tab. 5 und 6). Männer mit einem Vermeidungsziel suchten signifikant häufiger eine Nachsorgeambulanz auf. Beim Vergleich von Personen mit passiven/automatischen Selbstregulationsstrategien und Personen mit aktiven/expliziten Selbstregulationsstrategien ergab sich hinsichtlich der Häufigkeit psychiatrischer Diagnosen sowie des statischen Rückfallrisikos hingegen kein signifikanter Unterschied (Tab. 5 und 6).

\section{Diskussion}

In der vorliegenden Untersuchung wurde das Selbstregulationsmodell sexueller Delinquenz und Rückfälligkeit (SRM; Ward und Hudson 1998, 2000) erstmalig in einer deutschsprachigen Stichprobe von Personen, die aufgrund eines Kindesmissbrauchsdelikts verurteilt wurden, in Bezug auf verschiedene forensische, klinische und kriminologische
Merkmale untersucht. In Anlehnung an die theoretischen Grundlagen des SRM wurde die Hypothese aufgestellt, dass Probanden mit einem Annäherungsziel eine höhere Rückfallwahrscheinlichkeit aufweisen als Probanden mit einem Vermeidungsziel. Auch wenn weniger deutlich ausgeprägt als in früheren Untersuchungen, konnten Männer, die aufgrund eines Kindesmissbrauchsdelikts verurteilt wurden, häufiger einem Annäherungs- als einem Vermeidungsziel zugeordnet werden (Bickley und Beech 2002; Kingston et al. 2012). Ebenso vergleichbar zu früheren Studien wies ungefähr die Hälfte der Männer der aktuellen Stichprobe passive/automatische Selbstregulationsmechanismen auf, während die andere Hälfte aktive/explizite Selbstregulationsmechanismen zeigte (Bickley und Beech 2002; Kingston et al. 2012).

Zunächst zeigten die Ergebnisse, dass Probanden, die dem vermeidend-aktiven Tatbegehungspfad zugeordnet werden konnten, eine bessere Schulausbildung aufwiesen, im Vergleich zu Personen aus dem vermeidend-passiven Pfad. Nach den theoretischen Annahmen des SRM verfügen Personen aus dem vermeidend-aktiven Pfad zwar über geeignete Selbstregulationsstrategien, allerdings ist die Auswahl einer geeigneten Selbstregulationsstrategie in 
emotional fordernden Situationen häufig defizitär. Demgegenüber weisen Probanden des vermeidend-passiven Pfads fehlende Selbstregulationsfähigkeiten auf, sodass es bei emotionaler Belastung eher zu straffälligem Verhalten kommt. Zwar lässt sich das Ausmaß der Schulausbildung nicht mit der Intelligenz einer Person gleichsetzen, dennoch ergibt sich diesbezüglich ein hoher Zusammenhang (Donati et al. 2019). In früheren Untersuchungen konnte gezeigt werden, dass das Ausmaß der individuellen Selbstkontrolle sowohl positiv mit dem IQ als auch mit der Einsatzbereitschaft in der Schule korreliert (Eisenberg et al. 2010; Shamosh und Gray 2007), wodurch der in unserer Stichprobe gefundene Zusammenhang erklärt werden könnte.

Ebenso wie auch in früheren Untersuchungen wiesen Probanden mit einem Annäherungsziel hypothesenkonform in der aktuellen Untersuchung einen höheren Static-99-Wert und somit ein höheres Rückfallrisiko auf, im Vergleich zu Probanden mit einem Vermeidungsziel (z. B. Kingston et al. 2012). Entsprechend dem ,Risk-need-responsivity“(RNR)Modell (Andrews et al. 2011) besteht bei Personen mit einem Annäherungsziel folglich auch ein erhöhter Therapiebedarf. Kritisch ist dies jedoch insbesondere vor dem Hintergrund, dass Probanden mit einem Annäherungsziel eine geringere Ansprechbarkeit bezüglich psychotherapeutischer, risikosenkender Interventionen aufweisen (Webster 2005). Darüber hinaus wiesen Personen mit Annäherungszielen auch häufiger eine Pädophiliediagnose auf, weshalb bei dieser Gruppe angenommen werden könnte, dass eine besonders intensive Behandlung, insbesondere der sexuell devianten Interessen, angezeigt wäre, die - sofern die entsprechenden somatischen und psychologischen Voraussetzungen erfüllt sind - auch medikamentöse Behandlungsoptionen beinhalten könnte (Turner und Briken 2018a, 2018b; Sauter et al. 2020).

Entsprechend dem erhöhten Risiko für die Begehung einer erneuten Sexualstraftat wurden Probanden mit einem Annäherungsziel im Schnitt auch zu längeren Freiheitsstrafen verurteilt und befanden sich zum Zeitpunkt der Untersuchung häufiger im intramuralen Setting (d.h. in geschlossenen Einrichtungen des Maßregel- und Strafvollzugs). Trotz des höheren Risikos und des damit einhergehenden erhöhten Therapiebedarfs sowie der höheren Rate an pädosexuellen Störungen waren Personen mit einem Annäherungsziel häufiger im Strafvollzug zu finden, im Vergleich zu Probanden mit einem Vermeidungsziel. Dies könnte vor dem Hintergrund, dass die therapeutischen Angebote im Strafvollzug geringer ausfallen werden als im Maßregelvollzug, und den Annahmen des RNR-Modells, dass bei dieser Personengruppe eigentlich ein besonders intensives Interventionsprogramm angezeigt wäre, kritisch beurteilt werden. Demgegenüber fanden sich Probanden mit einem Vermei- dungsziel häufiger in ambulanter Behandlung, was eine Folge des niedrigeren Basisrisikos sein könnte.

Bei der Unterteilung der eingeschlossenen Probanden bezüglich der Selbstregulationsstrategien zeigten sich keine Unterschiede zwischen den beiden Gruppen, weder in Bezug auf die psychiatrischen Diagnosen noch in Bezug auf das individuelle Rückfallrisiko, obwohl auch hier, basierend auf früheren empirischen Untersuchungen (z.B. Kingston et al. 2013) sowie aufgrund theoretischer Annahmen (Yates et al. 2009), ein höheres Rückfallrisiko bei Personen mit aktiven/expliziten Strategien hätte erwartet werden können. Da die Stichprobengröße der vorliegenden Studie relativ gering ausfiel und damit die Subgruppeneinteilung zwangsläufig Limitationen unterlag, sollten der fehlende Gruppenunterschied in der vorliegenden Studie mit Zurückhaltung interpretiert und die Ergebnisse weiterer Untersuchungen mit größeren Stichproben abgewartet werden. In zukünftigen Untersuchungen wäre es wünschenswert, die Probanden nicht nur bezüglich der Ziele und Selbstregulationsstrategien einzuteilen, sondern alle 4 von Ward und Hudson (1998, 2000) vorgeschlagenen Tatbegehungspfade in den Analysen zu berücksichtigen. Dies würde eine differenziertere Auswertung erlauben und möglicherweise zur Bildung noch homogenerer Untergruppen führen, die wiederum nötig sind, um individualisierte therapeutische Interventionen $\mathrm{zu}$ entwickeln. Relevant ist darüber hinaus insbesondere der Effekt der geringeren therapeutischen Ansprechbarkeit von Personen mit Annäherungszielen, da diese gleichzeitig einen höheren Therapiebedarf nach dem RNR-Modell (Andrews et al. 2011) aufweisen. Sollte sich die geringere therapeutische Ansprechbarkeit in zukünftigen Studien bestätigen, hätte dies unmittelbare klinische und forensische Implikationen für die Behandlung und Begutachtung dieser Probanden. In einem solchen Fall wäre es entscheidend, die Faktoren zu identifizieren, die zu der geringeren therapeutischen Ansprechbarkeit führen, damit diese gezielt bearbeitet werden könnten.

Zusammenfassend liefern die Ergebnisse der vorliegenden Untersuchung erste Hinweise darauf, dass es mithilfe theoretisch fundierter Modelle gelingen kann, die ursprünglich sehr heterogene Gruppe von Personen, die aufgrund eines Kindesmissbrauchsdelikts verurteilt wurden, zu strukturieren, wodurch die angewandten Interventionen besser auf die individuellen Motive und Bedürfnisse der Probanden angepasst werden können. Die gefundenen Unterschiede waren jedoch weniger deutlich ausgeprägt als erwartet, wodurch sich Fragen nach der theoretischen Präzision der bislang formulierten Modellparameter stellen. Darüber hinaus sollten im Rahmen zukünftiger Forschungsbemühungen hinsichtlich des SRM auch verstärkt die messtheoretischen und diagnostischen Aspekte diskutiert werden, um die Möglichkeiten der reliablen Erfassung der SRM-bezogenen Konstrukte weiter zu erhöhen. 
Funding Open Access funding provided by Projekt DEAL.

Interessenkonflikt P.G. Hertz, M. Rettenberger, R. Welsch und D. Turner geben an, dass kein Interessenkonflikt besteht.

Open Access Dieser Artikel wird unter der Creative Commons Namensnennung 4.0 International Lizenz veröffentlicht, welche die Nutzung, Vervielfältigung, Bearbeitung, Verbreitung und Wiedergabe in jeglichem Medium und Format erlaubt, sofern Sie den/die ursprünglichen Autor(en) und die Quelle ordnungsgemäß nennen, einen Link zur Creative Commons Lizenz beifügen und angeben, ob Änderungen vorgenommen wurden.

Die in diesem Artikel enthaltenen Bilder und sonstiges Drittmaterial unterliegen ebenfalls der genannten Creative Commons Lizenz, sofern sich aus der Abbildungslegende nichts anderes ergibt. Sofern das betreffende Material nicht unter der genannten Creative Commons Lizenz steht und die betreffende Handlung nicht nach gesetzlichen Vorschriften erlaubt ist, ist für die oben aufgeführten Weiterverwendungen des Materials die Einwilligung des jeweiligen Rechteinhabers einzuholen.

Weitere Details zur Lizenz entnehmen Sie bitte der Lizenzinformation auf http://creativecommons.org/licenses/by/4.0/deed.de.

\section{Literatur}

Andrews DA, Bonta J, Wormith JS (2011) The risk-need-responsivity (RNR) model. Crim Justice Behav 38(7):735-755

Bickley JA, Beech AR (2002) An investigation of the Ward and Hudson pathways model of the sexual offense process with child abusers. J Interpers Violence 17(4):371-393

Donati G, Dumontheil I, Meaburn EL (2019) Genome-wide association study of latent cognitive measures in adolescence: genetic overlap with intelligence and education. Mind Brain Educ 13(3):224-233

Eisenberg N, Valiente C, Eggum ND (2010) Self-regulation and school readiness. Early Educ Dev 21(5):681-698

Gotch K, Carter M, Stotler-Turner E (2007) Relationship between the self-regulation model and level of psychopathy in a sample of sex offenders. Paper presented at the Annual Convention of the Association for the Treatment of Sexual Abusers, San Diego

Hanson RK, Harris AJ (2001) A structured approach to evaluating change among sexual offenders. Sex Abuse 13(2):105-122

Hanson RK, Thornton D (1999) Static-99: Improving actuarial risk assessments for sex offenders. User report 99-02. Department of the Solicitor General of Canada, Ottawa

Hanson RK, Thornton D (2000) Improving risk assessments for sex offenders: a comparison of three actuarial scales. Law Hum Behav 24(1):119-136

Kingston DA, Yates PM, Firestone P (2012) The self-regulation model of sexual offending: relationship to risk and need. Law Hum Behav 36(3):215-224

Kingston DA, Yates PM, Olver ME (2013) The self-regulation model of sexual offending: intermediate outcomes and posttreatment reoffending. Sex Abuse 26(5):1-21. https://doi.org/10.1177/ 1079063213495896

Kingston DA, Yates PM, Simons D (2009) The self-regulation model of sexual offender treatment: relationship to risk and the good lives model. Paper presented at the Annual Research and Treatment Conference for the Association for the Treatment of Sexual Abusers, Dallas

Leguizamo A, Harris DA, Lambine ME (2010) The self-regulation model of sexual offending: Validation and relationship to offender type and criminal versatility/specialization. Paper presented at the
Annual Research and Treatment Conference for the Association for the Treatment of Sexual Abusers, Phoenix

Marshall WL (1996) Assessment, treatment, and theorizing about sex offenders: developments during the past twenty years and future directions. Crim Justice Behav 23(1):162-199

Rettenberger M, Eher R (2006) Die deutsche Übersetzung des Static 99 zur aktuarischen Kriminalprognose verurteilter Sexualstraftäter. Monatsschr Kriminol Strafrechtsreform 89(1):352-365

Rettenberger M, Matthes A, Schilling F, Eher R (2011) Die Validität dynamisch-veränderbarer Risikofaktoren bei der Vorhersage einschlägiger Rückfälle pädosexueller Straftäter. Forens Psychiatr Psychol Kriminol 5(1):45-53

Sauter J, Turner D, Briken P, Rettenberger M (2020) Testosterone-lowering medication and its association with recidivism risk in individuals convicted of sexual offenses. Sex Abuse. https://doi.org/ 10.1177/1079063220910723

Shamosh NA, Gray JR (2007) The relation between fluid intelligence and self-regulatory depletion. Cogn Emot 21(8):1833-1843

Simons D, McCullar B, Tyler C (2008) Evaluation of the good lives model approach to treatment planning. Paper presented at the annual research and treatment conference of the Association for the Treatment of Sexual Abusers, Atlanta

Turner D, Briken P (2018a) Treatment of paraphilic disorders in sexual offenders or men with a risk of sexual offending with luteinizing hormone-releasing hormone agonists: An updated systematic review. J Sex Med 15(1):77-93

Turner D, Briken P (2018b) Die Behandlung paraphiler Störungen mit GnRH-Agonisten unter besonderer Berücksichtigung ethischer und rechtlicher Aspekte. Forens Psychiatr Psychol Kriminol 12(4):344-351

Turner D, Rettenberger M (2020) Neuropsychological functioning in child sexual abusers: A systematic review. Aggres Violent Behav. https://doi.org/10.1016/j.avb.2020.101405

Turner D, Laier C, Brand M, Bockshammer T, Welsch R, Rettenberger M (2018) Response inhibition and impulsive decisionmaking in sexual offenders against children. $\mathbf{J}$ Abnorm Psychol 127(5):471-481

Ward T, Hudson SM (1998) A model of the relapse process in sexual offenders. J Interpers Violence 13(6):700-725

Ward T, Hudson SM (2000) A self-regulation model of relapse prevention. In: Laws D, Hudson SM, Ward T (Hrsg) Remaking relapse prevention with sex offenders: a sourcebook. SAGE, Thousand Oaks, S 79-10 https://doi.org/10.4135/9781452224954.n5

Ward T, Louden K, Hudson SM, Marshall WL (1995) A descriptive model of the offence process. J Interpers Violence 10:452-472

Webster SD (2005) Pathways to sexual offense recidivism following treatment: an examination of the ward and hudson self-regulation model of relapse. J Interpers Violence 20(10):1175-1196

Welsch R, Schmidt AF, Turner D, Rettenberger M (2020) Test-retest reliability and temporal agreement of direct and indirect sexual interest measures. Sex Abuse. https://doi.org/10.1177/ 1079063220904354

Yates PM, Kingston DA (2006) The self-regulation model of sexual offending: The relationship between offence pathways and static and dynamic sexual offence risk. Sex Abuse 18(3):259-270

Yates PM, Ward T (2007) Treatment of sexual offenders: Relapse prevention and beyond. In: Witkiewitz KA, Marlatt G (Hrsg) Therapist's guide to evidence-based relapse prevention. Elsevier, San Diego, S 215-234

Yates PM, Ward T (2008) Good lives, self-regulation, and risk management: an integrated model of sexual offender assessment and treatment. Sex Abus Aust N Z 1(1):3-20

Yates PM, Kingston DA, Ward T (2009) The self-regulation model of the offense and re-offense process: Volume III. Pacific Psychological Assessment Corporation, Victoria 